\title{
Altered hypothalamic functional connectivity in post-traumatic headache after mild traumatic brain injury
}

\author{
Liyan Lư ${ }^{\dagger}$, Fengfang Li ${ }^{\dagger}$, Peng Wang, Huiyou Chen, Yu-Chen Chen ${ }^{*}\left(\mathbb{D}\right.$ and Xindao Yin ${ }^{*}$
}

\begin{abstract}
Background: Post-traumatic headache (PTH) is one of the most frequent symptoms following mild traumatic brain injury (mTBI). Neuroimaging studies implicate hypothalamic function connectivity (FC) disruption as an important factor in pain disorders. However, it is unknown whether there are alterations in the hypothalamus-based resting state FC within PTH following $\mathrm{mTBI}$ at the acute stage and its relationship with headache symptom measurement.

Methods: Forty-four mTBI patients with PTH, 27 mTBI patients without PTH and 43 healthy controls who were well matched for age, gender, and years of education were enrolled in this study. All participants underwent restingstate functional magnetic resonance imaging (fMRI) scanning as well as headache symptom measurement and cognitive assessment. Hypothalamic resting state networks were characterized by using a standard seed-based whole-brain correlation method. The bilateral hypothalamic FC was compared among the three groups. Furthermore, the correlations between hypothalamic resting state networks and headache frequency, headache intensity and MoCA scores was investigated in mTBI patients with PTH using Pearson rank correlation.

Results: Compared with mTBI patients without PTH, mTBI patients with PTH at the acute stage presented significantly decreased left hypothalamus-based FC with the right middle frontal gyrus (MFG) and right medial superior frontal gyrus (mSFG), and significantly decreased right hypothalamus-based FC with the right MFG. Decreased FC of the right MFG was significantly positively associated with headache frequency and headache intensity $(r=0.339, p=0.024 ; r=0.408, p=0.006$, respectively). Decreased FC of the right mSFG was significantly positively associated with headache frequency and headache intensity $(r=0.740, p<0.0001 ; r=0.655, p<0.0001$, respectively).
\end{abstract}

Conclusion: Our data provided evidence of disrupted hypothalamic FC in patients with acute mTBI with PTH, while abnormal FC significantly correlated with headache symptom measurement. Taken together, these changes may play an essential role in the neuropathological mechanism of mTBI patients with PTH.

Keywords: Functional connectivity, Hypothalamus, Post-traumatic headache, Mild traumatic brain injury

\footnotetext{
*Correspondence: chenyuchen1989@126.com; y.163yy@163.com

${ }^{\dagger}$ Liyan Lu and Fengfang Li contributed equally to this work.

Department of Radiology, Nanjing First Hospital, Nanjing Medical University,

No.68, Changle Road, Nanjing 210006, China
}

C C The Author(s). 2020 Open Access This article is licensed under a Creative Commons Attribution 4.0 International License, which permits use, sharing, adaptation, distribution and reproduction in any medium or format, as long as you give appropriate credit to the original author(s) and the source, provide a link to the Creative Commons licence, and indicate if changes were made. The images or other third party material in this article are included in the article's Creative Commons licence, unless indicated otherwise in a credit line to the material. If material is not included in the article's Creative Commons licence and your intended use is not permitted by statutory regulation or exceeds the permitted use, you will need to obtain permission directly from the copyright holder. To view a copy of this licence, visit http://creativecommons.org/licenses/by/4.0/ The Creative Commons Public Domain Dedication waiver (http://creativecommons.org/publicdomain/zero/1.0/) applies to the data made available in this article, unless otherwise stated in a credit line to the data. 


\section{Background}

Traumatic brain injury (TBI) is a significant worldwide health problem with major effects on both morbidity and mortality [1]. Among all traumatic brain injuries, mild traumatic brain injury (mTBI) is highly prevalent [2]. Following mTBI, patients frequently suffer lifelong disabilities, including post-traumatic headache (PTH), depression, insomnia, dizziness and hypomnesia. PTH is the most common and persistent symptom, and almost $70 \%$ of individuals with mTBI report pain-related problems and complaints. According to the International Classification of Headache Disorders Third Edition (ICHD-3), PTH is considered as an "acute" headache disorder if it develops within 3 months after the injury and as a "persistent" condition if it continues for longer than 3 months [3]. Persistent post traumatic injury (PPTH) may sometimes be underestimated because many patients never go to the hospital to obtain headache medication, which ultimately leads to long-lasting disability and imposes major burdens on society. However, it is still challenging for us to identify patients with mTBI with PTH, since the conventional computed tomography (CT) and magnetic resonance imaging (MRI)) cannot identify brain abnormalities [4]. Therefore, the underlying pathophysiologic mechanisms of PTH following $\mathrm{mTBI}$ are poorly understood.

The main phenotype of PTH in patients with $\mathrm{mTBI}$ is consistent with that of migraine [5, 6], and PTH commonly has characteristics similar to migraine. Thus, there are shared underlying neurochemical mechanisms between migraine and PTH [7]. Several studies have shown that various brain structures, such as the brainstem, central dopaminergic system and hypothalamus, are involved in the pathophysiology of migraine [8-10]. In particular, recently, the hypothalamus has been suggested to play an important role in the generation of migraine attacks and the sustainment of migraine pain [8]. Furthermore, in migraine, the hypothalamus has been reported to be involved not only in different stages of the migraine cycle (interictal, preictal and ictal) and the pathophysiology of migraine chronification, but also in clinical features of migraine, including yawning, tiredness and mood changes [8]. How hypothalamic changes might result in migraine is more intriguing. Neuroimaging studies revealed structural and functional alterations of the hypothalamus within migraine. One recent study showed that the volume of the hypothalamus was significantly decreased in migraine patients, compared with controls and that the volume of the hypothalamus was negatively correlated with headache frequency [10]. Additionally, several studies using functional MRI (fMRI) have indicated that migraine patients had both increased hypothalamic functional connectivity (FC) with a number of brain regions, including the frontal, parietal, temporal, subcortical, brainstem and cerebellum regions, and decreased hypothalamic FC with frontal and occipital regions $[9,11]$. Given some overlapping pathophysiological features underlying migraine and $\mathrm{PTH}$, the hypothalamus is presumed to be an important biomarker for the diagnosis and treatment of PTH. Morphological differences (including multifocal lower gray matter, cortical thinning and volume loss and functional alterations regarding pain processing) have been shown in patients with PTH after mTBI. Dumkrieger et al. reported that headache frequency was significantly correlated with static FC of the right posterior insula with the left hypothalamus in PTH [12]. However, to date, no study directly focusing on the hypothalamic FC alterations has been conducted in PTH following mTBI.

Thus, the present study aimed to investigate the FC between the hypothalamus and other brain regions in mTBI patients with PTH and further explore the association between any functional brain abnormality and various clinical features of PTH. By doing so, we utimately aimed to detect the possible pathophysiologic mechanisms of the hypothalamus in PTH following acute mTBI.

\section{Methods \\ Subjects}

This study was approved by the Institutional Review Board of Nanjing Medical University. All subjects provided written informed consent before undergoing MRI.

Between December 2017 and January 2019, patients with a diagnosis of mTBI were prospectively enrolled in this study. mTBI was defined based on the American Congress of Rehabitation Medicine [13]. Inclusion criteria were as follows: (a) patients aged 18 or older; (b) loss of consciousness $<30 \mathrm{~min}$; (c) Glasgow Coma Score (GCS) of 13-15; and (d)post-traumatic amnesia $<24 \mathrm{~h}$. Exclusion criteria were as follows: (a) previous head injury; (b) history of pre-existing neurological or psychiatric disease; (c) history of illicit drug use or substance abuse; (d) dental appliances that might distort the functional MR images; (e) left-handedness; and (f) history of migraine or any other headache prior to injury. The healthy control participants were recruited through local advertisements who met the same exclusion criteria applied to the patient group.

\section{Headache symptom measurement}

After a duration of 12 months, two experienced headache specialists confirmed all headache diagnoses using International Classification of Headache Disorders 3rd edition, beta version (ICHD-3 beta) diagnostic criteria [3]. Participants with PTH provided detailed information about their headaches including predominant localization, headache frequency (days/months) and 
headache intensity. Participants completed the Visual Analog Scale (VAS) [14], a numeric rating scale ranging from 0 to 10 where 0 represents no pain and 10 represents the most severe pain imaginable to report their headache intensity.

\section{Cognitive evaluation}

Given the emergency care setting, it was not feasible to perform a full battery of cognitive assessments. Therefore, a short instrument called the Montreal Cognitive Assessment (MoCA) was used to assess the patients' neurocognitive status [15]. The MoCA is a sensitive cognitive screening test following $\mathrm{mTBI}$, and it only requires limited training to administer. The MoCA has been extensively used in patient with pain disorders and in the study of Santangelo et al. [16]. This test is administered in approximately $10 \mathrm{~min}$ and is scored on a maximum of 30 points. Scores greater than 26 were regarded as normal values, with lower scores indicating greater cognitive deficit. All participants completed the MoCA test within $48 \mathrm{~h}$ of MRI examination.

\section{Imaging acquisition}

A $3.0 \mathrm{~T}$ magnetic resonance imaging scanner (Ingenia, Philips Medical Systems, Netherlands) with an 8-channel head coil was used for this study. Functional images were obtained axially using a gradient echo-planar imaging sequence as follows: repetition time $(\mathrm{TR})=2000$ $\mathrm{ms}$; echo time $(\mathrm{TE})=30 \mathrm{~ms}$; slices $=36$; thickness $=4$ $\mathrm{mm}$; gap $=0 \mathrm{~mm}$; field of view $(\mathrm{FOV})=240 \mathrm{~mm} \times 240$ $\mathrm{mm}$; acquisition matrix $=64 \times 64$; and flip angle $(\mathrm{FA})=$ $90^{\circ}$. The fMRI sequence took $8 \mathrm{~min}$ and $8 \mathrm{~s}$. The threedimensional turbo fast-echo (3D-TFE) T1WI sequence had high resolution: $\mathrm{TR}=8.1 \mathrm{~ms} ; \mathrm{TE}=3.7 \mathrm{~ms}$; slices $=$ 170; thickness $=1 \mathrm{~mm}$; gap $=0 \mathrm{~mm}$; $F A=8^{\circ}$; acquisition matrix $=256 \times 256$; and $\mathrm{FOV}=256 \mathrm{~mm} \times 256 \mathrm{~mm}$; For fluid-attenuated inversion recovery (FLAIR), the specifications were as follows: $\mathrm{TR}=7000 \mathrm{~ms}$; $\mathrm{TE}=120 \mathrm{~ms}$; slices $=18$; slice thickness $=6 \mathrm{~mm}$; gap $=1.3 \mathrm{~mm} ; \mathrm{FA}=$ $110^{\circ}$; and voxel size $=0.65 \times 0.95 \times 6 \mathrm{~mm}^{3}$. The specifications for susceptibility weighted imaging (SWI) were as follows: $\mathrm{TR}=22 \mathrm{~mm} ; \mathrm{TE}=34 \mathrm{~ms} ; \mathrm{FA}=20 ;$ matrix $=$ $276 \times 319$; slice thickness $=1 \mathrm{~mm}$; and FOV $=220 \mathrm{~mm} \times$ $220 \mathrm{~mm}$. SWI used a 3D gradient echo (GRE) sequence. FLAIR and SWI were used to investigate the presence of traumatic lesions. Traumatic lesions could be shown by high signals on FLAIR and low signals on SWI.

\section{Image processing and analysis}

Functional image analyses were preprocessed with the toolbox Data Processing Assistant for Resting-State fMRI programs based on Statistical Parametric Mapping (SPM8, http://www.fil.ion.ucl.ac.uk/spm) and the resting-state fMRI data analysis toolkit (REST, http:// www.restfmri.net). The first 10 volumes were discarded and the remaining 230 consecutive volumes were used for data analysis. Afterwards, slice-timing adjustment and realignment for head motion correction were performed. Any participant who had a head motion greater than $3.0 \mathrm{~mm}$ or a rotation in the $\mathrm{x}, \mathrm{y}$, or $\mathrm{z}$ directions higher than $3.0^{\circ}$ were excluded. Data were spatially normalized to the Montreal Neurological Institute (MNI) template (resampling voxel size $=3 \times 3 \times 3 \mathrm{~mm}^{3}$ ) and smoothed with a Gaussian kernel of $6 \mathrm{~mm}$ full width at half maximum (FWHM) to increase the signal-to-noise ratio.

To examine the FC patterns for the hypothalamus, the seed ROIs of the left and right hypothalamus were generated using the WFU PickAtlas software. The mean time series of each ROI was acquired for the reference time course. Then, Pearson's correlation coefficients were computed between the mean signal change of each ROI and the time series of each voxel. Finally, a Fisher's z-transform was applied to improve the normality of the correlation coefficients. Differences in FC of the bilateral hypothalamus were compared between groups (mTBI vs. healthy controls; mTBI+PTH vs. mTBI-PTH; mTBI+ PTH vs. healthy controls; mTBI-PTH vs. healthy controls) using one-way analysis of variance (ANOVA) and subsequent $\mathrm{t}$-tests at a threshold of $p<0.001$ with multiple comparisons correction using the AlphaSim program (http://afni.nih.gov/afni/docpdf/AlphaSim.pdf) determined by Monte Carlo simulation (single voxel $p$ value $=0.001$, a minimum cluster size of 13 , within a GM mask corresponding to the AAL atlas) and controlling for age and gender.

\section{Statistical analyses}

Differences in demographic data among mTBI patients with PTH, mTBI patients without PTH and healthy controls were analyzed using one-way ANOVA. Furthermore, a post hoc test ( $t$-test for means and $\mathrm{X}^{2}$-test for proportions) was performed between mTBI patients with PTH and mTBI patients without PTH. $P<0.05$ was considered to be statistically significant.

To investigate the abnormal hypothalamic FC between mTBI patients with PTH, mTBI patients without PTH and healthy controls, one-way ANOVA and subsequent t-tests for each seed region were estimated. Then, surface-based cluster-wise correction for multiple comparisons was performed at the significance threshold of $p<0.001$ and the cluster-size threshold of $13 \mathrm{~mm}^{2}$, which was determined by Monte Carlo simulation (single voxel $p$ value $=0.001$, a minimum cluster size of 13 , within a GM mask corresponding to the AAL atlas). The correlation between fMRI data and headache characteristics (headache frequency and headache intensity) and between MoCA scores in participant cohorts was 
investigated by using Pearson's correlation analyses. We considered statistically significant Pearson's correlations with $p$ values lower than 0.05 .

\section{Results}

After follow-up evaluation, 71 patients with mTBI and 43 healthy controls were enrolled in this study. All the participants were divided into three groups: mTBI + PTH (patients with PTH after mTBI, $n=44$ ); mTBI PTH (patients without PTH after $\operatorname{mTBI}, n=27$ ); and healthy controls (healthy volunteers without any headache, $n=43$ ). Table 1 is a summary of the basic demographic characteristics of the mTBI + PTH group, mTBI - PTH group and healthy control group. These three groups are well-matched and did not show any significant differences in age $(p=0.624)$, gender $(p=1.000)$, years of education $(p=0.569)$ or MoCA $(p=0.300)$. No visible traumatic lesions were seen on conventional imaging such as FLAIR or SWI.

Whole patients with mTBI vs healthy controls Compared with healthy controls, all whole patients with mTBI demonstrated significantly reduced FC between the left hypothalamus and left fusiform gyrus, left Rolandic operculum, and right middle frontal gyrus (MFG) as well as reduced FC between the left hypothalamus and right medial superior frontal gyrus (mSFG) (Fig. 1, Table 2). All whole patients with mTBI exhibited significantly decreased FC between the right hypothalamus and right MFG and right postcentral gyrus as well as decreased FC between the right hypothalamus and left supplementary motor area relative to healthy controls (Fig. 1, Table 2).

mTBI patients with PTH vs healthy controls; mTBI patients without PTH

Compared with mTBI patients without PTH, mTBI patients with PTH showed a significant FC reduction between the left hypothalamus and right MSFG and right MFG as well as decreased FC between the right hypothalamus and right MFG (Fig. 2, Table 3).

mTBI patients with PTH vs healthy controls; mTBI patients without PTH vs healthy controls

Compared with healthy controls, mTBI patients without PTH and mTBI patients without PTH showed no significant difference, respectively.

\section{Correlation between the hypothalamic FC of identified regions and headache measurements}

In the mTBI + PTH group at the acute stage, headache frequency was significantly positively correlated with the hypoconnectivity in the right $\mathrm{mSFG}$ and right MFG $(r=$ $0.740, p<0.001 ; r=0.339, p=0.024$, respectively) (Fig. 3a and $\mathrm{c}$ ). Headache intensity was significantly positively correlated with decreased FC between the right mSFG and right MFG $(r=0.655, p<0.0001 ; r=0.408, p=0.006$, respectively) (Fig. $3 \mathrm{~b}$ and $\mathrm{d}$ ). There were no significant correlations between headache symptom measurements and the FC of other identified regions.

Table 1 Clinical and demographic data of the study population

\begin{tabular}{|c|c|c|c|c|}
\hline Characteristics & $\begin{array}{l}\text { mTBI + PTH } \\
(n=44)\end{array}$ & $\begin{array}{l}\text { mTBI-PTH } \\
(n=27)\end{array}$ & $\begin{array}{l}\mathrm{HCs} \\
(n=43)\end{array}$ & $\begin{array}{l}\text { Group comparison } \\
P \text { values }\end{array}$ \\
\hline Age (y) & $40.9 \pm 10.9$ & $40.5 \pm 10.6$ & $43.5 \pm 10.8$ & 0.624 \\
\hline Gender (male/female) & $17 / 27$ & $14 / 13$ & $17 / 26$ & 1.000 \\
\hline Education (y) & $11.8 \pm 3.5$ & $11.1 \pm 4.0$ & $12.7 \pm 2.9$ & 0.569 \\
\hline GCS Score & 15 & 15 & 15 & \\
\hline MoCA & $24.5 \pm 2.6$ & $24.4 \pm 3.2$ & $25.7 \pm 2.5$ & 0.300 \\
\hline \multicolumn{5}{|l|}{ Headache characteristics } \\
\hline \multicolumn{5}{|l|}{ Predominant side } \\
\hline Left & 17 & - & - & \\
\hline Right & 9 & - & - & \\
\hline Bilateral & 10 & - & - & \\
\hline Unilateral & 8 & - & - & \\
\hline Onset & $12.0 \pm 7.9$ & - & - & \\
\hline Headache frequency & $12.0 \pm 7.4$ & - & - & \\
\hline Headache intensity & $4.7 \pm 2.0$ & - & - & \\
\hline
\end{tabular}

Data are the mean \pm standard deviation; mTBI mild traumatic brain injury; PTH post-traumatic headache; HCs healthy controls; GCS Glasgow Coma Scale; MoCA Montreal Cognitive Assessment 


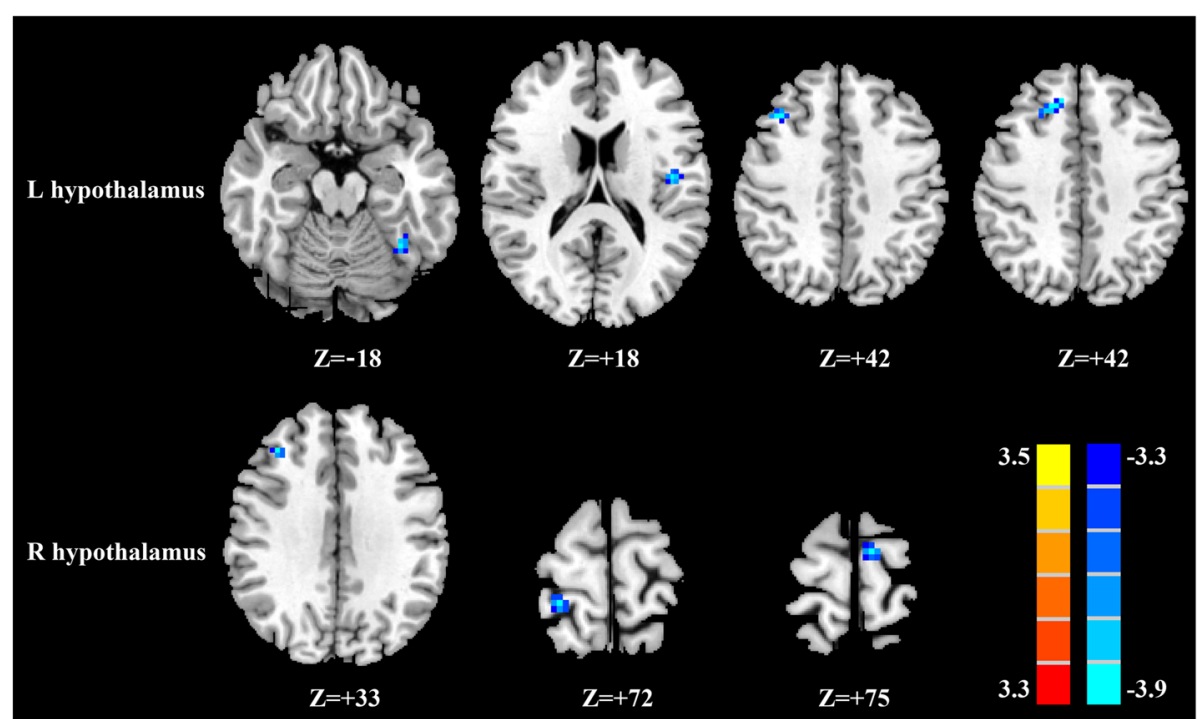

Fig. 1 Significant differences in the hypothalamic functional connectivity between patients with mTBI and healthy controls. From the left hypothalamus to the whole brain regions (top row); from the right hypothalamus to the whole brain regions (bottom row). Thresholds were set at a corrected $p<0.001$, determined by Monte Carlo simulation

\section{Discussion}

mTBI can cause injury to the hypothalamus through damage to hypothalamic cell bodies. One study demonstrated both microstructural and functional connectivity abnormalities of the hypothalamus in mTBI patients by using diffusional kurtosis imaging (DKI) and resting state fMRI (rs-fMRI) [17]. The main finding in the present study was the significantly decreased FC between the hypothalamus and frontal gyrus in patients with mTBI with PTH compared with patients with mTBI without PTH. These decreased FC alterations were significantly correlated with the headache frequency and headache intensity. Frontal regions as part of the brain network are involved in pain processing including the affective and cognitive evaluation of pain.
Notably, areas within the frontal lobe have previously been found to have abnormal structure, function, and FC in individuals with various pain disorders including PTH, migraine, cluster headache, and medication overuse headache $[5,18-20]$. Several other studies have identified relationships between headaches and structural and functional brain alterations in the frontal gyrus [2].

Of interest, we found that the FC between the left hypothalamus and right MFG and that between the right hypothalamus and right MFG were significantly decreased in patients with mTBI with PTH compared with patients with mTBI without PTH. This reduced FC between the left and right hypothalamus and right MFG was significantly positively correlated with headache frequency and headache intensity, respectively. The MFG,

Table 2 Significant brain regions showing altered hypothalamus functional connectivity in mTBI patients compared with healthy controls

\begin{tabular}{|c|c|c|c|c|}
\hline Brain region & BA & $\begin{array}{l}\text { Peak MNI coordinates } \\
x, y, z(m m)\end{array}$ & t value & Voxels \\
\hline \multicolumn{5}{|l|}{ L hypothalamus } \\
\hline L fusiform gyrus & 37 & $-36,-54,-18$ & -4.1003 & 17 \\
\hline L Rolandic operculum & 45 & $-45,-12,18$ & -4.1043 & 48 \\
\hline $\mathrm{R}$ middle frontal gyrus & 9 & $36,24,42$ & -3.8419 & 36 \\
\hline R medial superior frontal gyrus & 10 & $12,33,42$ & -3.7560 & 27 \\
\hline \multicolumn{5}{|l|}{ R hypothalamus } \\
\hline R middle frontal gyrus & 9 & $36,33,33$ & -4.6247 & 17 \\
\hline R postcentral gyrus & 2 & $24,-39,72$ & -4.6080 & 23 \\
\hline L supplementary motor area & 6 & $-9,-12,75$ & -4.4478 & 18 \\
\hline
\end{tabular}

A corrected threshold of $p<0.001$ determined by Monte Carlo simulation was taken as measuring that there was significant difference between groups. $B A$ Brodmann area; MNI Montreal Neurological Institute; $L$ left; $R$ right; $m T B I$ mild traumatic brain injury 


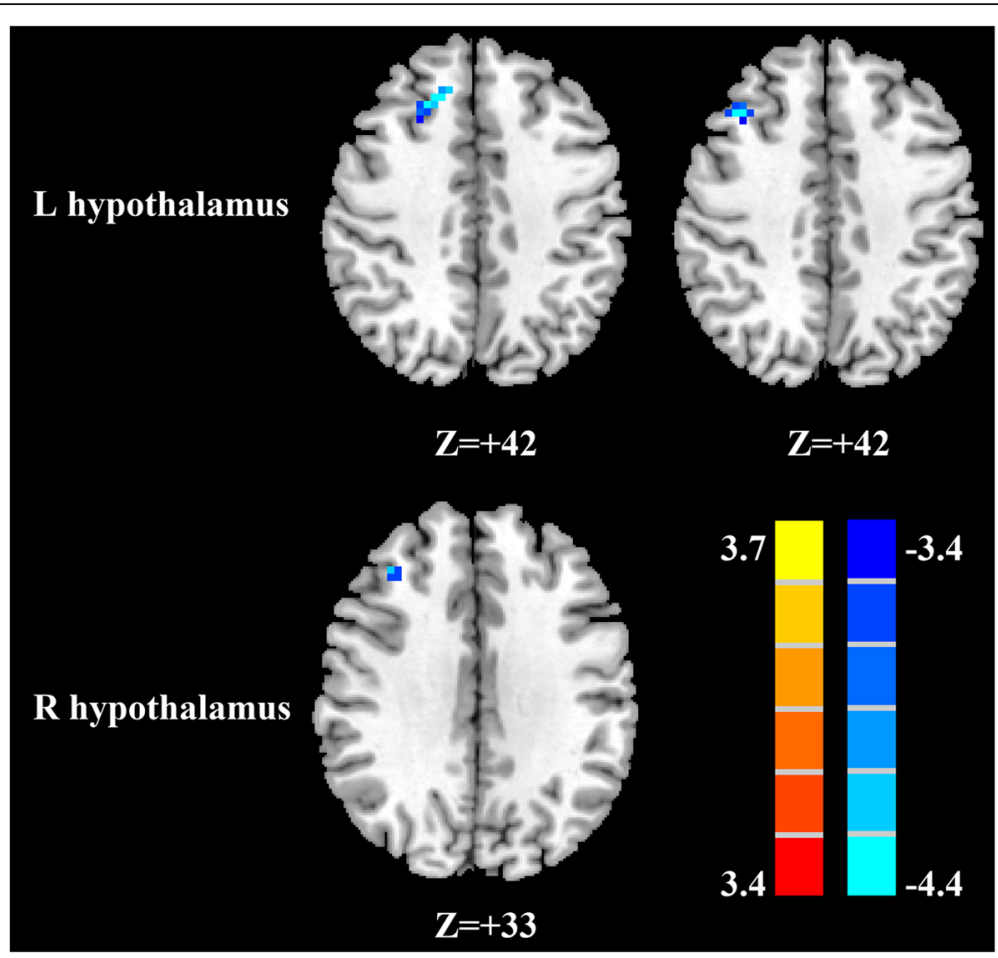

Fig. 2 Significant differences in the hypothalamic functional connectivity between patients with mTBI with PTH and patients with mTBI without PTH. (A) From the left hypothalamus to the whole brain regions (top row); from the right hypothalamus to the whole brain regions (bottom row). Thresholds were set at a corrected $p<0.001$, determined by Monte Carlo simulation

as part of the prefrontal cortex that regulates pain perception [21], has been reportedly involved in both dorsal and ventral attentional networks, responses to fearful expressions, emotional management and the detection of salient behaviorally relevant but task-irrelevant stimuli in patients with migraine compared with controls [22]. In particular, the MFG is considered to play an important role in the cognitive evaluation and modulation of pain. Both abnormal neurostructural and functional alterations in the MFG have been reported in patients with PTH relative to healthy controls in previous studies [2, 23]. Structurally, patients with PTH showed decreased structural integrity (smaller surface area, volume or thickness, or higher curvature) in the MFG. In addition, one recent voxel-based morphometric (VBM) study showed that the decreased volume in the left MFG is significantly correlated with disease duration [24-26]. Functionally, a study by Dumkrieger and colleagues examined static and dynamic FC of 59 regions of interest involved in pain processing and found dynamic FC alterations in the left and right MFG in patients with PTH [12]. From this perspective, our results were consistent with those of researchers $[27,28]$. The reason might be explained by the fact that impaired FC related to the MFG might diminish the capacity for pain evaluation and modulation.

We also demonstrated the significantly reduced FC between the left hypothalamus and right mSFG, and this decreased FC was significantly positively correlated with the headache frequency and headache intensity. The SFG region belongs to Brodmann area 10 (BA 10), which

Table 3 Significant brain regions showing altered hypothalamus functional connectivity in mTBI patients with PTH compared with mTBI patients without PTH

\begin{tabular}{|c|c|c|c|c|}
\hline Brain region & BA & Peak MNI coordinates $x, y, z(m m)$ & $\mathrm{t}$ value & Voxels \\
\hline \multicolumn{5}{|l|}{ L hypothalamus } \\
\hline R superior medial frontal gyrus & 10 & $18,30,42$ & -3.8630 & 34 \\
\hline R middle frontal gyrus & 9 & $39,24,42$ & -4.0160 & 22 \\
\hline \multicolumn{5}{|l|}{$\mathrm{R}$ hypothalamus } \\
\hline R middle frontal gyrus & 9 & $36,33,33$ & -4.3566 & 15 \\
\hline
\end{tabular}

A corrected threshold of $p<0.001$ determined by Monte Carlo simulation was taken as measuring that there was significant difference between groups. $B A$ Brodmann area; $M N I$ Montreal Neurological Institute; $L$ left; $R$ right; $m T B I$ mild traumatic brain injury; $P T H$ post traumatic headache 


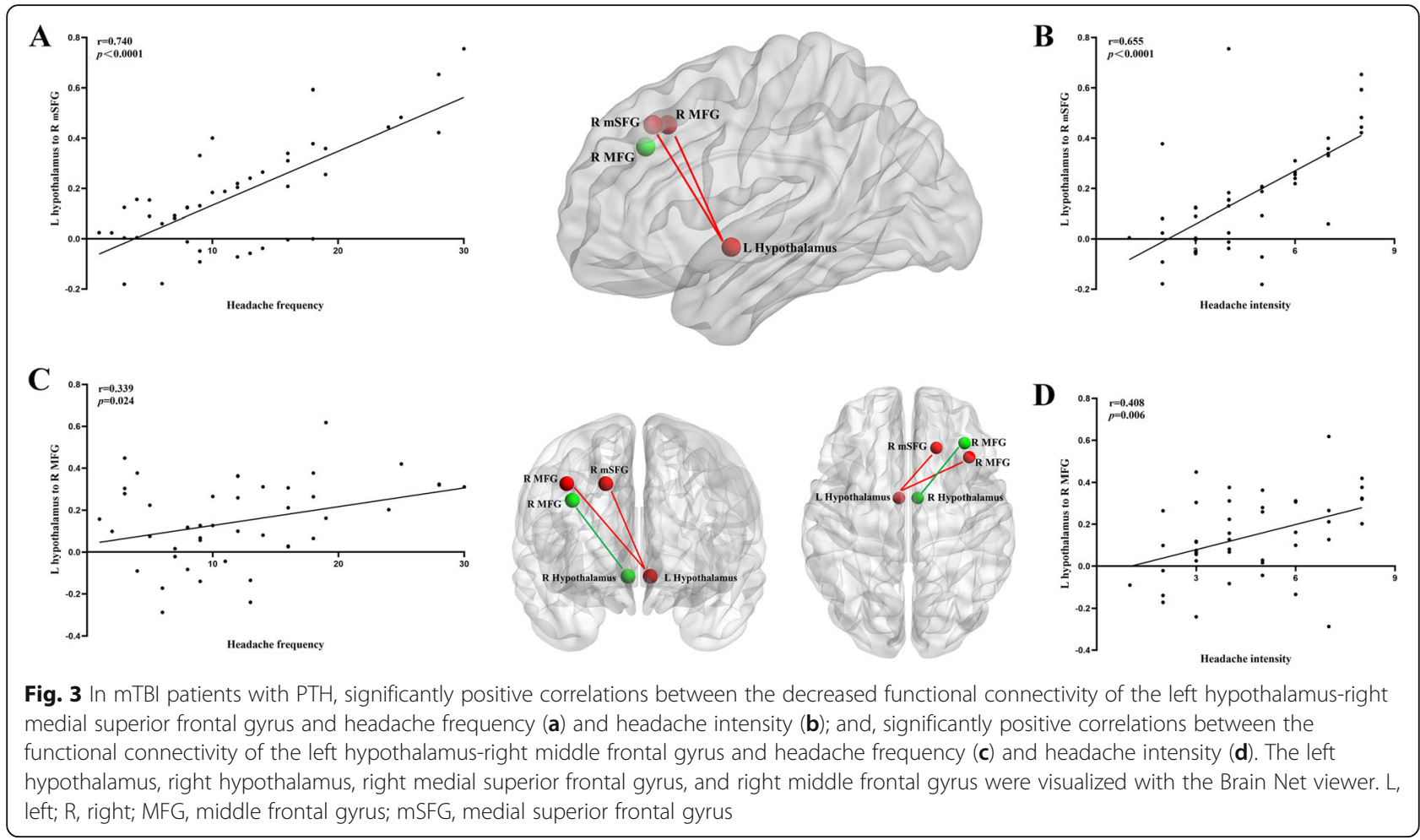

is the largest frontal brain region and is known as the anterior prefrontal cortex, frontopolar prefrontal cortex, or rostral prefrontal cortex. The cytoarchitecture of BA10 is characterized by more dendritic spines, a higher spine density, and a lower cell body density compared with other areas of the cortex, suggesting that BA 10 is more likely to accomplish these tasks of information integration [29]. In fact, BA 10 has been implicated in multiple integrative roles, including working memory, abstract reasoning, decision making, behavior control, nociceptive processing, and perceptual metacognition. Thus, the SFG, as one of the rich-club regions of BA 10, may have been involved in a great number of connections may lead to the modulation of cortical and subcortical nociceptive pathways. Compared with healthy controls, patients with PTH had less cortical thickness in the left and right SFG and demonstrated a negative correlation between left and right SFG thickness and headache frequency [2]. In addition, patients with pain disorders showed significant functional alterations, such as lower regional homogeneity ( $\mathrm{ReHo}$ ) and FC abnormalities in the superior frontal gyrus [24, 30]. More specifically, the medial frontal gyrus is known as the pain anticipation and modulation area through the anterior cingulate cortex (ACC), a key region of the pain matrix [30]. Sarmento et al. reported reduced N-acetylaspartate (NAA)/creatinine and increased choline/creatinine in the medial frontal lobe in patients with PTH compared with controls [31]. Our data of decreased FC between the left hypothalamus and right mSFG in mTBI patients with $\mathrm{PTH}$ are consistent with previous findings [32]. In the literature, this reduced FC may result in a permissive brain condition and the initiation of headache attacks, ultimately reflecting an insufficient pain modulating capacity.

Interestingly, our study demonstrated significant FC reduction between the left hypothalamus and contralateral MFG and mSFG. This asymmetry in connectivity during the resting state is small but consistent [33]. In the present study, hemispheric dominance may potentially play a role in defining the areas injured.

In the current study, we further showed that decreased FC between the left hypothalamus and left fusiform gyrus, left Rolandic operculum, right MFG and right mSFG as well as decreased FC between the right hypothalamus and right MFG, right postcentral gyrus and left supplementary motor area in mTBI patients compared with healthy controls. Among these brain regions, the fusiform is involved in nociception/antinociception and neurocognitive aspects of pain processing [34, 35]. In $\mathrm{PTH}$, functional connectivity altered in the fusiform gyrus [12]. Evidence suggests that increased Rolandic operculum activation is correlated with some anticipatory processes. However, the exact role of the Rolandic operculum in pain disorders is unclear. fMRI studies have revealed activation of postcentral regions in response to painful stimulation [36]. Although no structural or functional alterations in the postcentral gyrus 
were found in PTH studies, cortical thinning, decreased amplitude of low-frequency fluctuation, hypoperfusion and FC abnormalities were shown in the postcentral gyrus in migraineurs [28]. The supplementary motor area, a region involved in motor planning and preparation, is activated in response to painful stimuli. Decreased supplementary motor area activation in patients with mTBI with PTH might indicate patients' response to avoid movement during pain because physical movement worsens the pain [37, 38]. Nevertheless, these four brain regions were not significantly related to the hypothalamus in patients with mTBI with PTH. This may be attributed to the small samples of participants and requires further work to investigate why these brain areas are not involved in PTH.

Our study has several limitations. First, our research has a cross-sectional design with a small sample size. Second, the different parts of the hypothalamus have distinct roles; the anterior part seems to play an important role in headache attack generation and headache chronification, while the posterior part of the hypothalamus seems to be important for the acute headache stage. However, the entire hypothalamus, rather than hypothalamus subregions, was examined in this study. The current study could be extended to the hypothalamic subdivisions to investigate the FC patterns of mTBI patients with PTH. Third, scales to measure neuropsychological state, including depression and anxiety during a headache, were not measured; hence, the influence of these neuropsychological factors on hypothalamic FC was not assessed. Finally, the phenotype of PTH was not classified in detail, and different phenotypes may influence hypothalamic FC.

\section{Conclusion}

In summary, this study revealed hypothalamic functional disconnections in mTBI patients with PTH compared to mTBI patients without PTH and healthy controls. There were associations between the reduced $\mathrm{FC}$ of hypothalamic-related brain regions and headache frequency and headache intensity. These results together could provide a new perspective to understanding the neuropathological mechanism underlying the PTH following acute mTBI to determine more appropriate management.

\footnotetext{
Abbreviations

mTBI: Mild traumatic brain injury; PTH: Post traumatic headache; ICHD3: International Classification of Headache Disorder Third Edition;

$\mathrm{CT}$ : Computed tomography; fMRI: Functional magnetic resonance imaging; FC: Functional connectivity; GCS: Glasgow Coma Score; VAS: Visual Analog Scale; MoCA: Montreal Cognitive Assessment; MNI: Montreal Neurological Institute; MFG: Middle frontal gyrus; mSFG: Medial superior frontal gyrus; VBM: Voxel-based morphometric; GMV: Gray matter volume; FOV: Field of view; FLAIR: Fluid-attenuated inversion recovery; SWI: Susceptibility weighted imaging; GRE: Gradient echo
}

\section{Acknowledgements}

We thank all the patients and controls for their participation in the study.

\section{Authors' contributions}

$\mathrm{LL}$ and $\mathrm{FL}$ designed the experiment, collected the data, performed the analysis, and wrote the paper. PW and $\mathrm{HC}$ helped collect the data and perform the analysis. XY and YC C contributed to the discussion and manuscript revision. All authors read and approved the final manuscript.

\section{Funding}

This study was supported by 333 High-level Talents Training Project of Jiangsu Province (No. BRA2019122) and Nanjing Medical University grant (No, 2017NJMU123)

\section{Availability of data and materials}

Clinical, neuroimaging and statistical data will be available upon request from any qualified investigator.

\section{Ethics approval and consent to participate}

The study protocol was approved by the Research Ethics Committee of the Nanjing Medical University prior to study initiation. All the subjects provided written informed consent before any study procedures.

\section{Consent for publication}

Not applicable.

\section{Competing interests}

The authors declare that they have no competing interests.

Received: 26 May 2020 Accepted: 22 July 2020

Published online: 28 July 2020

\section{References}

1. Niu X et al (2019) Disruption of periaqueductal grey-default mode network functional connectivity predicts persistent post-traumatic headache in mild traumatic brain injury. J Neurol Neurosurg Psychiatry 90(3):326-332

2. Schwedt TJ (2019) Structural and Functional Brain Alterations in Posttraumatic Headache Attributed to Mild Traumatic Brain Injury: A Narrative Review. Front Neurol 10:615

3. Headache Classification Committee of the International Headache Society (IHS) (2018) The International Classification of Headache Disorders, 3rd edition. Cephalalgia 38(1):1-211

4. Kurca E, Sivak S, Kucera P (2006) Impaired cognitive functions in mild traumatic brain injury patients with normal and pathologic magnetic resonance imaging. Neuroradiology 48(9):661-669

5. Schwedt TJ et al (2017) Persistent post-traumatic headache vs. migraine: an MRI study demonstrating differences in brain structure. J Headache Pain 18(1):017-0796

6. Packard RC (1999) Epidemiology and pathogenesis of posttraumatic headache. J Head Trauma Rehabil 14(1):9-21

7. Capi M et al (2020) Persistent Post-Traumatic Headache and Migraine: PreClinical Comparisons. Int J Environ Res Public Health 17:7

8. Schulte LH, Allers A, May A (2017) Hypothalamus as a mediator of chronic migraine: evidence from high-resolution fMRI. Neurology 88(21):2011-2016

9. Coppola G et al (2020) Increased neural connectivity between the hypothalamus and cortical resting-state functional networks in chronic migraine. J Neurol 267(1):185-191

10. Chen Z et al (2019) Volume of Hypothalamus as a Diagnostic Biomarker of Chronic Migraine. Front Neurol 10:606

11. Skorobogatykh K et al (2019) Functional connectivity studies in migraine: what have we learned? J Headache Pain 20(1):019-1047

12. Dumkrieger $G$ et al (2019) Static and dynamic functional connectivity differences between migraine and persistent post-traumatic headache: a resting-state magnetic resonance imaging study. Cephalalgia 39(11):13661381

13. Carroll L J et al (2004) Methodological issues and research recommendations for mild traumatic brain injury: the WHO collaborating Centre task force on mild traumatic brain injury. J Rehabil Med 43:113-125

14. Varndell W, Fry M, Elliott D (2017) A systematic review of observational pain assessment instruments for use with nonverbal intubated critically ill adult 
patients in the emergency department: an assessment of their suitability and psychometric properties. J Clin Nurs 26(1-2):7-32

15. de Guise E et al (2014) The Montreal cognitive assessment in persons with traumatic brain injury. Appl Neuropsychol Adult 21(2):128-135

16. Santangelo $\mathrm{G}$ et al (2016) Cognitive dysfunctions and psychological symptoms in migraine without aura: a cross-sectional study. J Headache Pain 17(1):016-0667

17. Zhou Y (2017) Abnormal structural and functional hypothalamic connectivity in mild traumatic brain injury. J Magn Reson Imaging 45(4): $1105-1112$

18. Jin C et al (2013) Structural and functional abnormalities in migraine patients without aura. NMR Biomed 26(1):58-64

19. Yang FC et al (2015) Altered hypothalamic functional connectivity in cluster headache: a longitudinal resting-state functional MRI study. J Neurol Neurosurg Psychiatry 86(4):437-445

20. Messina R, Filippi M, Goadsby PJ (2018) Recent advances in headache neuroimaging. Curr Opin Neurol 31(4):379-385

21. Chen $C$ et al (2019) Alterations in regional homogeneity assessed by fMRI in patients with migraine without Aura. J Med Syst 43(9):019-1425

22. Szabo $E$ et al (2019) Association between migraine frequency and neura response to emotional faces: an fMRI study. Neuroimage Clin 22(101790):26

23. Tessitore A et al (2013) Disrupted default mode network connectivity in migraine without aura. J Headache Pain 14(1):1129-2377

24. Wang $P$ et al (2014) Regional homogeneity abnormalities in patients with tension-type headache: a resting-state fMRI study. Neurosci Bull 30(6):949955

25. Absinta M et al (2012) Selective decreased grey matter volume of the painmatrix network in cluster headache. Cephalalgia 32(2):109-115

26. Magon $\mathrm{S}$ et al (2019) Cortical abnormalities in episodic migraine: A multicenter 3T MRI study. Cephalalgia 39(5):665-673

27. Ha SY, Park KM (2019) Alterations of structural connectivity in episodic cluster headache: a graph theoretical analysis. J Clin Neurosci 62:60-65

28. Huang $X$ et al (2019) Altered functional connectivity of the red nucleus and substantia nigra in migraine without aura. J Headache Pain 20(1):019-1058

29. Su J et al (2019) Relationship between changes in resting-state spontaneous brain activity and cognitive impairment in patients with CADASIL. J Headache Pain 20(1):019-0982

30. Yang FC et al (2013) Altered gray matter volume in the frontal pain modulation network in patients with cluster headache. Pain 154(6):801-807

31. Sarmento E et al (2009) Proton spectroscopy in patients with post-traumatic headache attributed to mild head injury. Headache 49(9):1345-1352

32. Russo A et al (2012) Executive resting-state network connectivity in migraine without aura. Cephalalgia 32(14):1041-1048

33. Raemaekers $\mathrm{M}$ et al (2018) Knowing left from right: asymmetric functional connectivity during resting state. Brain Struct Funct 223(4):1909-1922

34. Petrusic I et al (2018) Migraine with Aura: surface-based analysis of the cerebral cortex with magnetic resonance imaging. Korean J Radiol 19(4): 767-776

35. Bonanno $L$ et al (2020) Brain morphologic abnormalities in migraine patients: an observational study. J Headache Pain 21(1):020-01109

36. Kocsel $\mathrm{N}$ et al (2017) Trait Rumination Influences Neural Correlates of the Anticipation but Not the Consumption Phase of Reward Processing. Front Behav Neurosci 11:85

37. Schwedt TJ et al (2014) Enhanced pain-induced activity of pain-processing regions in a case-control study of episodic migraine. Cephalalgia 34(12): 947-958

38. Solstrand Dahlberg L et al (2018) Responsivity of Periaqueductal Gray Connectivity Is Related to Headache Frequency in Episodic Migraine. Front Neurol 9:61

\section{Publisher's Note}

Springer Nature remains neutral with regard to jurisdictional claims in published maps and institutional affiliations.

Ready to submit your research? Choose BMC and benefit from:

- fast, convenient online submission

- thorough peer review by experienced researchers in your field

- rapid publication on acceptance

- support for research data, including large and complex data types

- gold Open Access which fosters wider collaboration and increased citations

- maximum visibility for your research: over $100 \mathrm{M}$ website views per year

At $\mathrm{BMC}$, research is always in progress.

Learn more biomedcentral.com/submissions 